\title{
Cellphone evolution - applying evolution theory to an info-communication system
}

\author{
T. Faragó1,a , Á. Miklósi11,2,b \\ ${ }^{1}$ MTA-ELTE Comparative Ethological Research Group, Budapest, Hungary \\ 2 Department of Ethology, Eötvös Loránd University, Budapest, Hungary \\ ${ }^{a}$ mustela.nivalis@gmail.com \\ bamiklosi62@gmail.com
}

\begin{abstract}
In this paper we propose the application of evolutionary principles in studying technological devices and processes. First we introduce the concepts of Darwinian evolution, which we use to draw an analogy with technology and show how human-made devices can be treated as evolving artificial agents. After explaining the methodology of reconstructing evolutionary history, we apply successfully the delineated principles and methodology to an infocommunication system: cellphones. By modeling a possible phylogenetic tree of mobile phones and exploring technological changes through time, we are able to show evolutionary trends and parallels with biological processes.
\end{abstract}

\section{I.INTRODUCTION}

\section{A. Evolution of artificial systems}

There is a scientific consensus that the rich diversity of recent living organisms originated from past forms through an evolutionary process [1], [2]. The beauty of Darwin's idea is its simplicity: only a handful of principles must be satisfied in a system before a system is capable of evolving [3]

\section{1) Principles of evolution}

Let's take a system that contains (1) a limited amount of resources and (2) an initial population of agents in which (3) the resources are required for the agents to reproduce themselves. Because of the limitation of resources, the population will expand until it reaches the threshold when the agents will compete for the available resources. If the agents have attributes that determine their resourceexploitation ability (4) and they are able to transmit these only in an imperfect way to the next generation (5), then an evolutionary process will emerge [3].

The random changes that appear through the imperfect transmission of attributes will provide the basis for differences in the success of the agents to exploit resources and reproduce (this is called "fitness" in evolutionary biology). From generation to generation, some attributes, which are beneficial for the agents' survival, will spread and supplant other attributes. This process is called natural selection. In a biological system, the environment is manifested in different ecological niches, and various numbers of individuals with manifold attributes will try to enter these niches and maximize their fitness. These preconditions can inhere not just in biological but artificial systems, and these principles can be interpreted in domains of computation, technology [4], robotics [5], culture [6] and innovation [7].

\section{2) Evolution in technology}

First-world civilizations are extremely dependent on technology. There is a vast demand on new innovations and technological devices, and understanding how and why one or another product becomes successful is interesting both from a scientific and an economic point of view [8].

One possible approach is to view technological devices as evolving agents that are formed by selective pressures in the economy [9]. To explore this possibility, first we must explain how evolutionary principles can be applied and interpreted in the context of technological progress

\section{a) Initial population}

The subjects of selection and evolutionary processes are the technological devices produced by different companies. These devices bear numerous different technological innovations, and their specifications and appearances (design) determine their functionality. If a brand new device appears on the market, it has the potential to spread without change until new competitors emerge.

\section{b) Environment with limited resources}

The market can be interpreted as the limiting environment, and consumers who are willing to buy new devices represent the limited resources. In a narrow timeframe, this limited number of people will determine which device succeeds because they choose particular functions and designs based on their expectations, actual technical trends, and fashion. Note also that there is not a single unified market but also local markets ("niches") in which different products may have different potential to survive (e.g., "businessmen", "teenagers", etc.).

\section{c) Emergence of diversity}

The companies are interested in keeping up the demand for their product and engaging new consumers, so they will develop newer and newer versions with improved functionality and additional new innovations. In parallel, other companies will attempt to break into the market with similar devices. There will be devices with similar 
functionality on the market but with marked differences. The similar functionality can be achieved by using analogous technology or sometimes by copying the genuinely original devices. This 'transfer' of attributes is always imperfect due to avoidance of legal issues (e.g., patent rights) or continuous attempts to improve.

\section{d) Competition}

Different companies selling similar products with similar functions compete for the available resources, the consumers. Newer and newer devices emerge, and successful ones will spread and outgrow others. The changes and innovations are always based on earlier variants like in biological systems.

\section{B. Studying evolutionary changes}

One possible approach to study the forces that formed the agents during the course of evolution is to compare the agents' attributes and try to classify them by their degree of similarity [10]. This can be easily understood if we adopt the concept of the common ancestor.

\section{1) The phylogenetic tree}

From the logic of the evolution, it follows that more similar agents are more closely related. New attributes can appear only from earlier ones; thus more changes and differences appear in each generation. If we examine a group of different agents and group them hierarchically by their similarities, we can draw a phylogenetic tree representing how the different agents diverged from earlier forms [11]. The leaves of this tree represent the agents; branches with dichotomous divergences show the emergence of new agents; and the root, where all the braches run into each other, represents the hypothetical common ancestor, from which all the recent agents can be derived. This method has been extremely useful in biology when modeling phylogenetic relationships among extinct and extant species.

\section{2) Reconstructing the tree}

Hierarchical classification offers a useful method to reconstruct the possible lineage of the agents [12], and it gives a hint about the genetic relationship between the agents [13]. This method compares numerous attributes (characters) of the agents and organizes the data into a matrix. The characters can be coded as binary, categorical or continuous measures. Binary characters show the presence or absence of a given trait; the categorical characteristics are used when a trait shows several different states; and continuous characters can be any measured traits (e.g., weight).

This data matrix is transformed into a distance matrix representing the Euclidean distance of each character pair. From these distances we construct the hierarchy of our agents by the means of different methods. For example, the Unweighted Pair Group Method with Arithmetic Mean (UPGMA) groups cyclically the closest agents into a cluster and calculates this group's distance from the other agents by giving the mean distance between all the agents in the group [14], [15].

These methods construct numerous potential trees, and the researcher must choose the one that reflects the most probable actual evolutionary pathways of the studied agents [16]. First, one needs to set the root of the trees. Accordingly, we must define an outgroup that carries the most ancient traits and separated from our agents at the earliest time in evolution [17]. This way, one can determine the first branch and obtain the initial point of the tree. Next we assume that the most probable phylogenetic tree carries the smallest number of character changes (parsimony); thus the quality of any generated tree can be measured by its length counted in sum character changes.

Due to the large number of possible trees, heuristic search methods are used to find the most parsimonious tree [18]. E.g., bootstrapping or rearranging trees by Subtree Pruning and Regrafting (SPR) cuts off parts of the original tree, reattaches it to a different location repeatedly, calculates the character changes for each iteration and uses the most parsimonious tree for the next cycle [19].

\section{II.AIM}

In our study we attempt to apply the principles of evolution to an info-communication system, the mobile phone. Mobile communication devices have become an important part of human society and everyday life [20]. Their high prevalence in modern society, fast technological improvement and rich diversity make them especially good candidates for our evolutionary study. Moreover, studying their changes over time can provide interesting insight into the process of technological development and how it interacts with the users' tastes and expectations.

Here, we consider mobile phones as evolvable agents and draw an analogy to biological systems. We have collected data about the devices of the oldest mobile company (Nokia), from the earliest model to the models released in 2010. This approach provides an extensive picture of the history of cellphone evolution from the earliest stage to the era of smartphones.

\section{III.MATERIALS AND METHODS}

\section{B. Data collection}

The phones included in our analysis were chosen semirandomly to represent each year, series and category (e.g., low-end and high-end devices) in a balanced way. In other respects, the sampling was completely random. The technical data of the chosen models were collected from different open web databases (http://www.gsmarena.com; http://pdadb.net/index.php). We coded the technological features (118) in binary (76), categorical (17) or continuous (25) characters. The characters belonged to the following categories:

$\cdot$

- $\quad$ Network 


$\begin{array}{ll}\text { - } & \text { Frequency capability } \\ \text { - } & \text { Messaging } \\ \text { - } & \text { Internet connection } \\ \text { - } & \text { Services } \\ \text { - } & \text { Hardware } \\ \text { - } & \text { Coftware } \\ \text { - } & \text { Connera } \\ \text { - } & \text { Radio } \\ \text { - } & \text { Signaling } \\ \text { - } & \text { Accumulator } \\ \text { - } & \text { Dimensions }\end{array}$

The categorical characters could have 3 to 19 possible states (e.g., the technology built into the screen, USB type), while the continuous characters were metric attributes such as battery capacity or weight. Some characters were not definable such as camera details in cameraless devices; these were marked as non-applicable characters, and the tree-drawing algorithm ignored them. In several cases we found no data for some characters (e.g., the weight of old models), and these were treated as unknown. We set up a group of characters with ordered transitions allowing only step-by-step changes in both directions, while Form, Antenna and Primary/Secondary keyboard were set as unordered characters.

\section{Drawing the phylogenetic tree}

For calculating the distance matrix and drawing the possible trees, we used a Java-based modular cladistics software package called Mesquite (v2.74) [21]. We applied the UPGMA method for drawing possible hierarchies, and the parsimony analysis was made with SPR based on all the measured categorical and binary characters. The oldest model (Nokia 100) was set up as an outgroup for rooting the tree. For the analysis of the most parsimonious tree, we projected the characters at the branches and observed the changes, and we also explored the hierarchies (clads) of the models.

\section{IV.RESULTS AND DISCUSSION}

\section{B. General description of the tree}

The length of the most parsimonious tree was 561 steps, which is relatively high due to the large number of observed characters and phone types (Fig. 1). There are some character returns that appeared due to the limited functionality of the used software. We cannot give transition rules to limit character changes to our model, which are obviously present in mobile evolution (e.g. when colored screens appeared, this character will not reverse to monochrome state in a later branch). This likely explains that the suboptimal tree is probably longer than the most parsimonious tree. But, the reconstructed pattern of the trait changes will be still good for our analysis: in our tree it is conspicuous that the models from the same series are grouped together. One good example is Communicator (9000) series, which builds up two clads.
The tree also reflects the temporal distribution of the models. In general the older outdated models sit closer to the root, while the modern, new series of high-end models (E, C, N and $\mathrm{X}$ ) appear at the top of the tree. Interestingly, the newer 1000 series is forming a clad neighbored by older models. This pattern arises because the series contains low-end, cheap models with limited feature sets.

\section{Character changes}

\section{1) Continuous characters}

\section{a) Dimensions}

An overall evolutionary trend of miniaturization is clear from the tree. Both the area, thickness, and weight of the phones show this pattern, which is probably caused by the selective pressure for mobility. The largest and heaviest phones sit near the root, although there is a prominent reversal at the emergence of smartphones. This phenomenon is not an artifact, but rather is due to the rise of touchscreens, which results in a new tradeoff between shrinking the size of the phone and the usability and sensitivity of the touch panel. This reversal is not so prominent in the thickness of the phones; it occurs in the earlier smartphone series (e.g., 7700, 7710, N70, N80). Probably in the later high-end models, thanks to the newer miniaturizing technologies, the more advanced hardware became even smaller and could be fitted into a slimmer case.

The Communicator series was far heavier than its contemporaries because the series consisted of businessclass models with high-end functionality but without sophisticated ergonomics. These phones represent a good example of an extinct group that was superseded by new species.

\section{b) Screen}

The screen resolution also shows a rising pattern through the course of evolution. One exception is the Communicator clad, in which the functions required a high-resolution screen. Also, in newer models, the appearance of the cameras coincides with the increase in resolution. This can be a good exemplar of evolutionarycorrelated traits.

\section{c) Power-handling}

The power handling of the phones can be measured by the stand-by time or talk time. This depends on two features: the capacity of the battery and the energy consumption of the technologies in the device. The capacities of the models are similar, but there are marked differences in endurance. The energy consumption of the phones shows a tessellated pattern: The earlier diverged models and the middle branches of series 5-6-7000 devices have relatively low endurance. In contrast, the low-end 1-2000 models and the high-end smartphones have a longer lifespan. This is probably due to the more advanced technologies and better power-handling of the smartphones, while on the other hand the low energy needs of the low-end devices. 


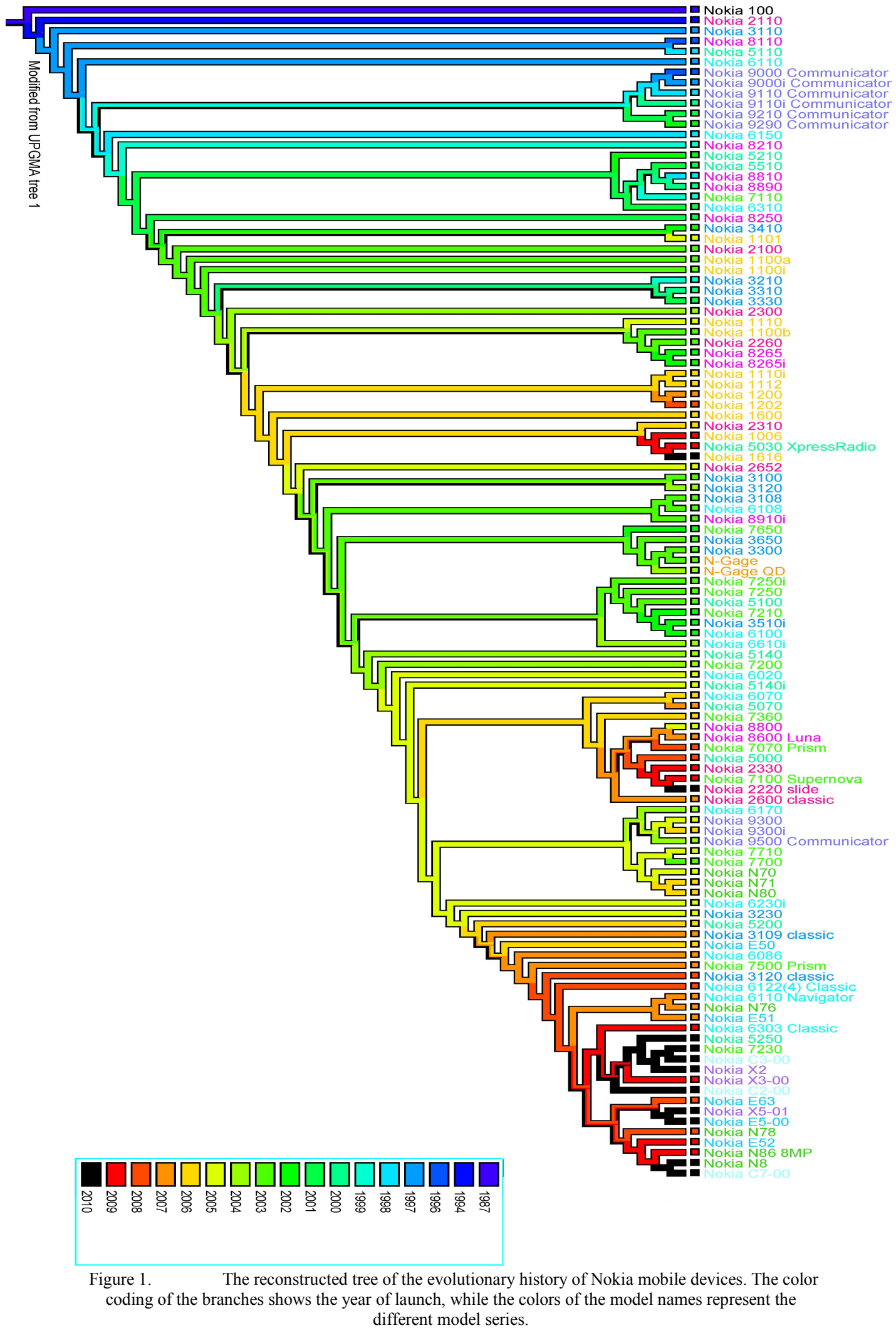

\section{1) Categorical character changes}

\section{a) Data communication}

While the outgroup Nokia 100 used the NMT-900 analogue data communication system, the models after 1990 use the digital GSM network. The GSM system allowed for text messaging and internet access, which opened a new niche for text-message capable devices. This process is analogous to adaptive radiation, when a species invades a new ecological environment and new resources become available depending on adaptive speciation by descendants of the ancestor. If subpopulations of the invading species adapt and 
specialize to the new circumstances, they diverge and become new species. There are two branches containing models that do not use GSM (1006, 2260, 8265 and $8265 \mathrm{i}$ ), because they were intended for the US market, where CDMA and TDMA networks are the standard.

Within the GSM network, three new consecutive data protocols emerged: GPRS, EDGE and 3G. These appeared on our tree in that order.

\section{b) Appearance}

The form and the design of the devices are undoubtedly important aspects from the users' point of view. The form of the device does not primarily reflect functionality, but often the form is constrained by technological limitations or economic considerations. New technological innovations might also allow for new design and form. Importantly, cultural factors including specific fashion trends might have an effect as well. The ancient form is the "candybar" (elongated form with buttons below the screen) derived from the old wired telephone receivers, and all the other forms can be derived from it. Newer forms reduce the size, hide the numeric or QWERTY keyboard, and protect the screen. This development is represented by the "clamshell" form of the Communicator clads, and also the "flip form" devices which are also grouped together $(3108,6108,8910 i)$. The N-Gage and the 3300 models with a horizontal candybar form were experimental models for gaming, which is more common in young people. Far from this clad appears the 5510 and the 7700 , both with the same form. While the former was designed for easy two-handed texting, the latter had a large touchscreen. These similar forms are a nice example of evolutionary convergence, where non-related species facing similar selective forces develop similar characteristics relying on different mechanisms (like the fins of fishes and dolphins). Interestingly, the "slider" cellphone form appears dispersed throughout all the different clads.

Another interesting morphological trait is the antenna, which is extendable in archaic form, becoming fixed later. Eventually it disappears permanently with the model 8210. This phenomenon is analogous to when an unnecessary organ or trait disappears. For example, several cave-living organisms lost their eyes during evolution because the energy cost to develop the whole organ is relatively high; therefore the eyeless individuals had an advantage. Similar processes occurred with cellphone keyboards as well. With the sweep of touchscreens, the buttons began to disappear.

\section{c) Signalling}

One basic function of a mobile phone is the signaling of incoming calls and messages. In archaic form, phones used monophonic tones, which disappeared when polyphonic tones appeared in the 2300 model. In the newest models at the upper part of the tree, this character is also swept out by the playback of wav and mp3sounds. Vibration is an adaptation for signaling in very noisy environments or in situations when loud ringtones can be disturbing. This feature appeared first in the 8210 model and is missing from only 3 later models. The two Communicator models lack this feature because the phone would need an especially strong buzzing engine, which was too costly and was possibly harmful to the hardware. Interestingly the missing vibration feature in the 3210 model is due to a random mutation: the engine was left out accidentally during the manufacturing. Despite this handicap, this model was a success thanks to its other progressive features.

\section{II.CONCLUSION}

Our aim was to study the development of an infocommunication device, the mobile phone. We showed that the principles of evolution can be applied to an artificial system. The methods of numerical taxonomy and cladistics, which had been developed for reconstructing evolutionary relationships, were applied successfully to the history of cellphones.

We reconstructed hypothetical relations of mobile phones and drew a possible phylogenetic tree. We identified evolutionary trends by studying changes in traits through the phylogenetic tree. For example, we showed that the process of miniaturization is counterbalanced by the tradeoff caused by the emergence of touchscreens and that the richness of functionalities brought elevated energy consumption, which in turn possibly led to the invention of lighter and more powerful batteries. As we saw, the evolution of mobile phones is formed by several different selective forces such as human culture [22]. In addition to the technological tradeoffs, the users have a strong effect on the changes in consumer goods. For example. the changes of Teddy bears' facial features gradually evolved from an initial state into one with infant-like features [23]. Similarly, musical tones can evolve from random sounds through artificial evolutionary process due to the selective pressure of the musical taste of listeners [7]. Slight changes in devices and tools can create highly variable population of agents competing with each other and their own ancestors [24]. We found that due to lower competitive abilities, several clads have become extinct (e.g., the Communicator series), similar to archaic words in languages or technological solutions that were outperformed by newer innovations [22].

Besides the high-end models, the simpler low-cost ones are also adaptive and found their own niche in the market as different populations can adapt to different environments. We can also draw a parallel here with the striking cultural differences found in different populations of humans [22].

\section{A. Limitations and outlook}

However, we are aware of the limitations of our work. Due to the technical limitations of the used tree generating software, we could not apply specially ordered character transitions. This caused clear mismatches on the tree, and we had to run the analysis with a less parsimonious tree that represents local minima in length. If we involved more devices form different companies, 
we would have been able to see how the competition among companies affects the evolutionary process. If we could add sales and consumer satisfaction data to our analysis, it is possible that we could provide a more elaborate analysis and point out trends that will determine the future market.

\section{ACKNOWLEDGMENT}

This study was supported by the ETOCOM project (TÁMOP-4.2.2-08/1/KMR-2008-0007) through the Hungarian National Development Agency in the framework of Social Renewal Operative Programme supported by the EU and co-financed by the European Social Fund and the Hungarian Academy of Sciences (F01/031). We are thankful to Julie Hecht for correcting our English.

\section{REFERENCES}

[1] R. Dawkins, The Blind Watchmaker: Why the Evidence of Evolution Reveals a Universe Without Design, 3rd ed. New York: W. W. Norton, 2005.

[2] C. Darwin, On the origin of species by means of natural selection, or the preservation of favoured races in the struggle for life. London, UK: John Murray, 1859.

[3] V. Csányi, "The replicative model of evolution: A general theory," World Futures, vol. 23, no. 1, pp. 31-65, Apr. 1987.

[4] G. Kampis and L. Gulyás, "Emergence out of interaction: Developing evolutionary technology for design innovation," Advanced Engineering Informatics, vol. 20, no. 3, pp. 313-320, Jul. 2006.

[5] J. C. Bongard, "Morphological change in machines accelerates the evolution of robust behavior," Proceedings of the National Academy of Sciences, vol. 108, no. 4, pp. 1234-1239, Jan. 2011.

[6] P. Steadman, The evolution of designs: biological analogy in architecture and the applied arts, 2nd ed. London, UK: Routledge, 2008.

[7] R. M. MacCallum, M. Mauch, A. Burt, and A. M. Leroi, "Evolution of music by public choice," Proceedings of the National Academy of Sciences, no. 7, Jun. 2012.

[8] U. Witt, "On the proper interpretation of 'evolution' in economics and its implications for production theory," Journal of Economic Methodology, vol. 11, no. 2, pp. 125-146, Jun. 2004.

[9] G. M. Hodgson, "Darwinism in economics: from analogy to ontology," Journal of Evolutionary Economics, vol. 12, no. 3, pp. 259-281, Jul. 2002.
[10] W. Hennig, "Phylogenetic Systematics," Annual Review of Entomology, vol. 10, no. 1, pp. 97-116, Jan. 1965.

[11] B. Dayrat, "Ancestor-descendant relationships and the reconstruction of the Tree of Life," Paleobiology, vol. 31, no. 3, pp. 347-353, 2005.

[12] J. Podani, "Taxonomy versus evolution," Taxon, vol. 58, no. November, pp. 1049-1053, 2009.

[13] A. D. Gordon, "A review of hierarchical classification," Journal of the Royal Statistical Society. Series A ( ..., vol. 150, no. 2, pp. 119137, 1987.

[14] R. R. Sokal and C. D. Michener, "A statistical method for evaluating systematic relationships," University of Kansas Science Bulletin, vol. 38, pp. 1409-1438, 1958.

[15] F. J. Rohlf, "Classification of Aedes by numerical taxonomic methods (Diptera: Culicidae)," Annals of the Entomological Society of America, vol. 56, 1963.

[16] A. Charig, "Cladistics: a different point of view," Biologist, 1981.

[17] L. E. Watrous and Q. D. Wheeler, "The outgroup comparison method of character analysis," Systematic zoology, vol. 30, no. 1, pp. 1-11, 1981.

[18] G. Giribet, "Efficient tree searches with available algorithms.," Evolutionary bioinformatics online, vol. 3, pp. 341-56, Jan. 2007.

[19] B. Efron, "Bootstrap methods: another look at the jackknife," The annals of Statistics, vol. 7, no. 1, pp. 1-26, 1979.

[20] L. Srivastava, "Mobile phones and the evolution of social behaviour," Behaviour \& Information Technology, vol. 24, no. 2, pp. 111-129, Mar. 2005.

[21] W. P. Maddison and D. R. Maddison, "Mesquite: a modular system for evolutionary analysis." 2010.

[22] A. Mesoudi, A. Whiten, and K. N. Laland, "Perspective: is human cultural evolution Darwinian? Evidence reviewed from the perspective of the Origin of Species.," Evolution; international journal of organic evolution, vol. 58, no. 1, pp. 1-11, Jan. 2004.

[23] R. A. Hinde and L. A. Barden, "The evolution of the teddy bear," Animal Behaviour, vol. 33, no. 4, pp. 1371-1373, Nov. 1985.

[24] G. Basalla, The Evolution of Technology Cambridge, UK: Cambridge University Press, 1989. 